\title{
Displaying Multiphase CT Angiography Using a Time-Variant Color Map: Practical Considerations and Potential Applications in Patients with Acute Stroke
}

\author{
(D).M. Ospel, (D) O. Volny, (D). Qiu, (D) M. Najm, (D) N. Kashani, D M. Goyal, and DB.K. Menon
}

\begin{abstract}
SUMMARY: Various imaging protocols exist for the identification of vessel occlusion and assessment of collateral flow in acute stroke. CT perfusion is particularly popular because the color maps are a striking visual indicator of pathology. Multiphase CTA has similar diagnostic and prognostic ability but requires more expertise to interpret. This article presents a new multiphase CTA display format that incorporates vascular information from all phases of the multiphase CTA series in a single time-variant color map, thereby facilitating multiphase CTA interpretation, particularly for less experienced readers. Exemplary cases of multiphase CTA from this new display format are compared with conventional multiphase CTA, CT perfusion, and follow-up imaging to demonstrate how time-variant multiphase CTA color maps facilitate assessment of collateral flow, detection of distal and multiple intracranial occlusions, differentiation of pseudo-occlusion from real occlusion, and assessment of flow relevance of stenoses, anteand retrograde flow patterns, and clot permeability.
\end{abstract}

ABBREVIATIONS: $\mathrm{ACA}=$ anterior cerebral artery; $\mathrm{mCTA}=$ multiphase $\mathrm{CT}$ angiography; $\mathrm{PCA}=$ posterior cerebral artery

S ince the publication of multiple recent trials attesting to the benefit of thrombolysis and thrombectomy even in patients presenting late after stroke onset, fast and reliable detection of cerebrovascular pathology is ever more important. Several ongoing trials are using imaging to further expand treatment indications in patients with acute stroke. Imaging protocols designed for patients with acute stroke, therefore, need to be reliable in detecting and measuring a whole host of cerebrovascular pathology, including arterial flow dynamics across time, pial

Received October 9, 2019; accepted after revision November 19. From the Division of Neuroradiology, Clinic of Radiology and Nuclear Medicine (J.M.O.), University Hospital Basel, University of Basel, Basel, Switzerland; Departments of Clinical Neurosciences (J.M.O., O.V., W.Q., M.N., M.G.) and Radiology (N.K., M.G.), University of Calgary, Calgary, Alberta, Canada; International Clinical Research Centre (O.V.), Stroke Research Program, St. Anne's University Hospital, Brno, Czech Republic; and Departments of Radiologyand Clinical Neurosciences, Radiology and Community Health Sciences (B.K.M.), Cumming School of Medicine, University of Calgary, Foothills Medical Centre, Calgary, Alberta, Canada.

Paper previously presented as an educational exhibit at: Annual Meeting of the Radiological Society of North America, December 1-6, 2019; Chicago, Illinois.

Please address correspondence to Bijoy K. Menon, MD, Departments of Radiology and Clinical Neurosciences, Radiology and Community Health Sciences, Cumming School of Medicine, University of Calgary, Foothills Medical Centre, 1403 29th St NW, Calgary, AB Canada T2N 2T9; e-mail: docbijoymenon@gmail.com; @johanna_ospel

\section{- Indicates open access to non-subscribers at www.ajnr.org}

Indicates article with supplemental on-line appendix and table.

Indicates article with supplemental on-line photos.

http://dx.doi.org/10.3174/ajnr.A6376 collateral status, distal arterial occlusions, and thrombus characteristics such as extent and permeability, carotid pseudo-occlusions, and venous thrombus, to name a few. Because acute stroke is a time-sensitive state in which any delay in decision-making affects patient outcome, such imaging protocols need to be easy to interpret for the average stroke physician in a small hospital. These imaging protocols also need to be easy to acquire and process without being affected too much by patient motion, a common occurrence when imaging patients with stroke.

Multiphase CTA (mCTA) provides time-resolved images of the cerebral vasculature that satisfy many of the above requirements for use in patients with acute stroke. The current display format of mCTA consists of 3 gray-scale images of the cerebral vasculature displayed side by side; visualization requires the reader to link these images together and scroll through them simultaneously. Interpretation, therefore, requires some degree of expertise. The technique was, however, used as a selection criterion for endovascular treatment in patients with acute ischemic stroke with large-vessel occlusions presenting $<12$ hours from symptom onset in the Endovascular Treatment for Small Core and Proximal Occlusion Ischemic Stroke (ESCAPE) trial and is being used in the ongoing Safety and Efficacy of NA-1 in Subjects Undergoing Endovascular Thrombectomy for Stroke (ESCAPENA1; NCT02930018) and the A Randomized Controlled Trial of TNK-tPA Versus Standard of Care for Minor Ischemic Stroke With Proven Occlusion (TEMPO 2; NCT02398656) trials. Good collaterals as determined on CTA have also been shown to 


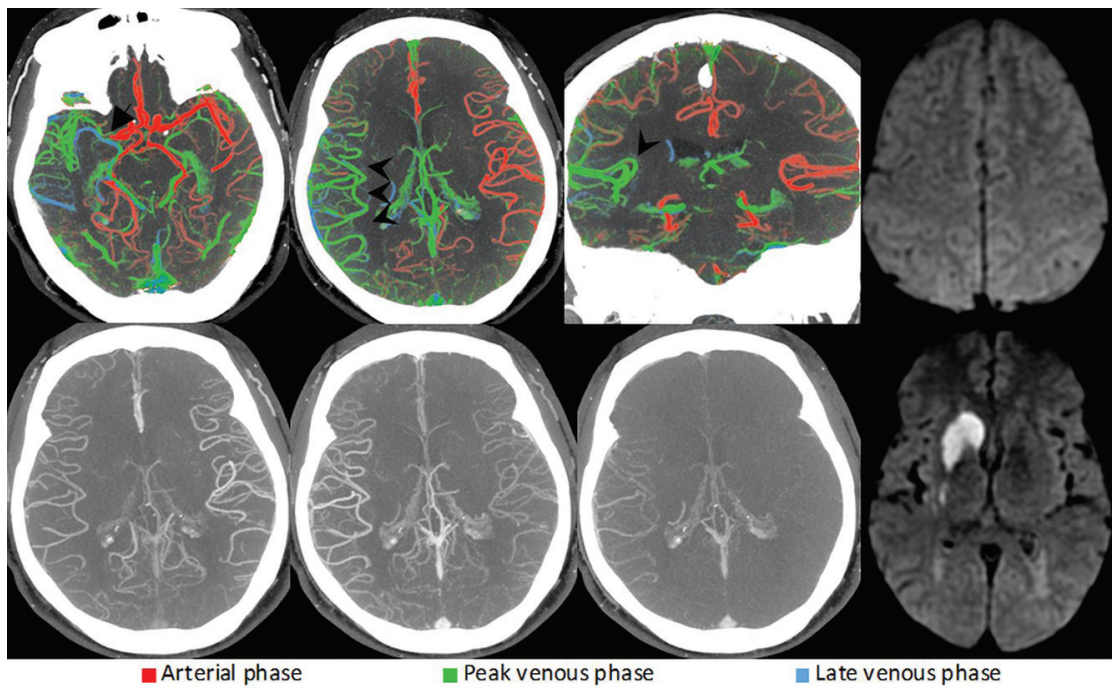

FIG 1. Right-sided M1 segment MCA occlusion (arrow). ColorViz summation maps (upper row) show predominantly green vessels in the affected territory (arrowheads), indicating a 1phase delay; The vessel extent is identical to that of the unaffected hemisphere. The findings are consistent with good pial artery filling and collateral flow. Corresponding conventional mCTA images are displayed in the lower row. The patient received intravenous alteplase, and follow-up MR imaging 24 hours after symptom onset (upper and lower right) shows infarction of the caudate head and lentiform nucleus only, with sparing of the cortex.

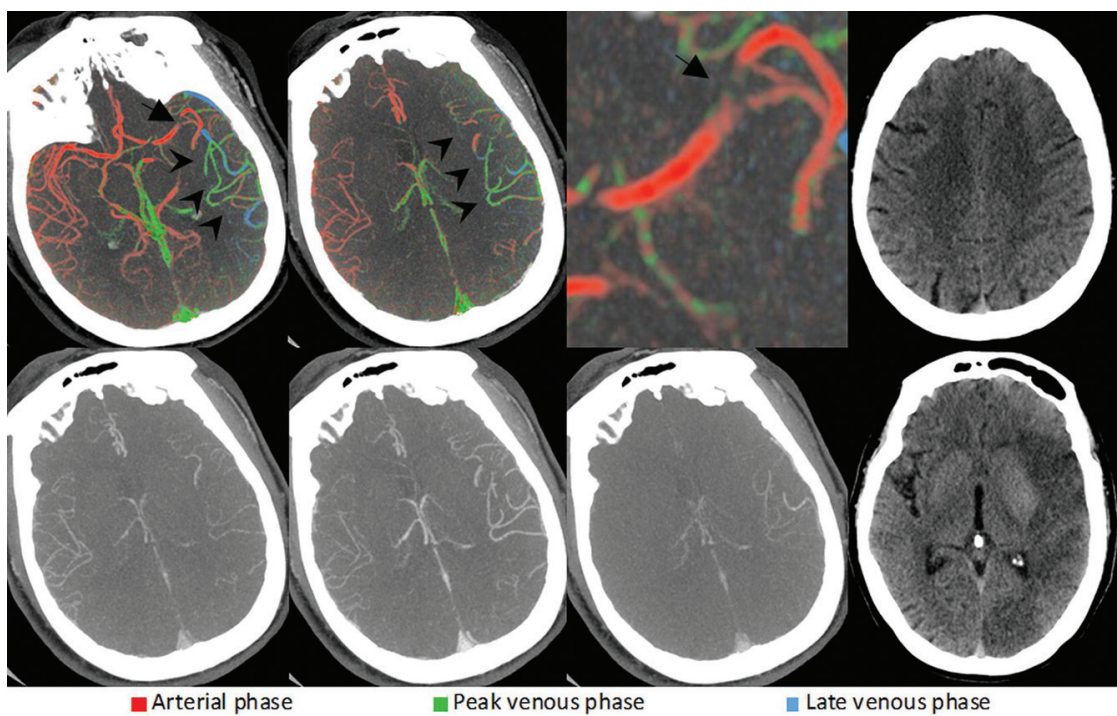

FIG 2. Left-sided M1 segment MCA occlusion (arrow). ColorViz summation maps (upper row) show predominantly green vessels (arrowheads) in the affected territory, indicating a 1-phase delay. However, the vessel extent is reduced compared with the unaffected hemisphere. Hence, the findings are consistent with intermediate pial artery filling and collateral flow. Corresponding conventional mCTA images are displayed in the lower row. The patient was treated with intravenous alteplase, and follow-up CT after 24 hours (upper and lower right) reveals an incomplete infarction pattern. While the insula, M1, and M2 regions are infarcted, the M3, M4, M5, and M6 regions are spared. Infarction is also present in the left PCA territory, indicating a fetal left PCA origin.

correlate with reduced infarct volume in patients presenting beyond 6 hours. ${ }^{1}$ Most interesting, no association between collateral status and outcome was noted in this study. ${ }^{1} \mathrm{CTP}$, another imaging technique with time-resolved images of parenchymal blood flow but with more complex acquisition and postprocessing, is considered easier to interpret because the display format is a single color-coded cerebral map of estimated blood flow and predicted tissue fate. This feature is in spite of obvious disadvantages of CTP, including longer image-acquisition times, susceptibility to patient motion, more radiation exposure, an additional contrast dose, a lack of whole-brain coverage, and complex and heterogeneous postprocessing algorithms. mCTA, on the other hand, requires no postprocessing, has a lower radiation dose, and can be acquired in $<30$ seconds (as opposed to CTP, which requires continuous scanning for 45-90 seconds), thereby minimizing motion artifacts.

In this primarily descriptive article, we present the case for a novel mCTA display format that encodes vascular information from all mCTA phases into a single color-coded map using a simple algorithm, thereby combining the indicator effect of color with the technical advantages of mCTA.

\section{Patient Cohort}

Imaging data were obtained are from the Precise and Rapid Assessment of Collaterals Using Multi-phase CTA in the Triage of Patients With Acute Ischemic Stroke for IA Therapy ${ }^{1}$ a prospective observational multicenter study that seeks to analyze the incremental value of MCTA and CTP in acute ischemic stroke $(n=596)$. The local institutional ethics boards (University of Calgary, Foothills Medical center) approved the study. Patient baseline characteristics from this study are summarized in the On-line Table.

\section{Image Acquisition and Interpretation}

The details about the multiphase CTA acquisition technique, parameters, and image interpretation can be found in the On-line Appendix. Color-coded mCTA summation maps presented here were created on a workstation using the FastStroke research prototype (GE Healthcare, Milwaukee, Wisconsin), a CT image-analysis software package that is intended to display the full set of imaging information of 


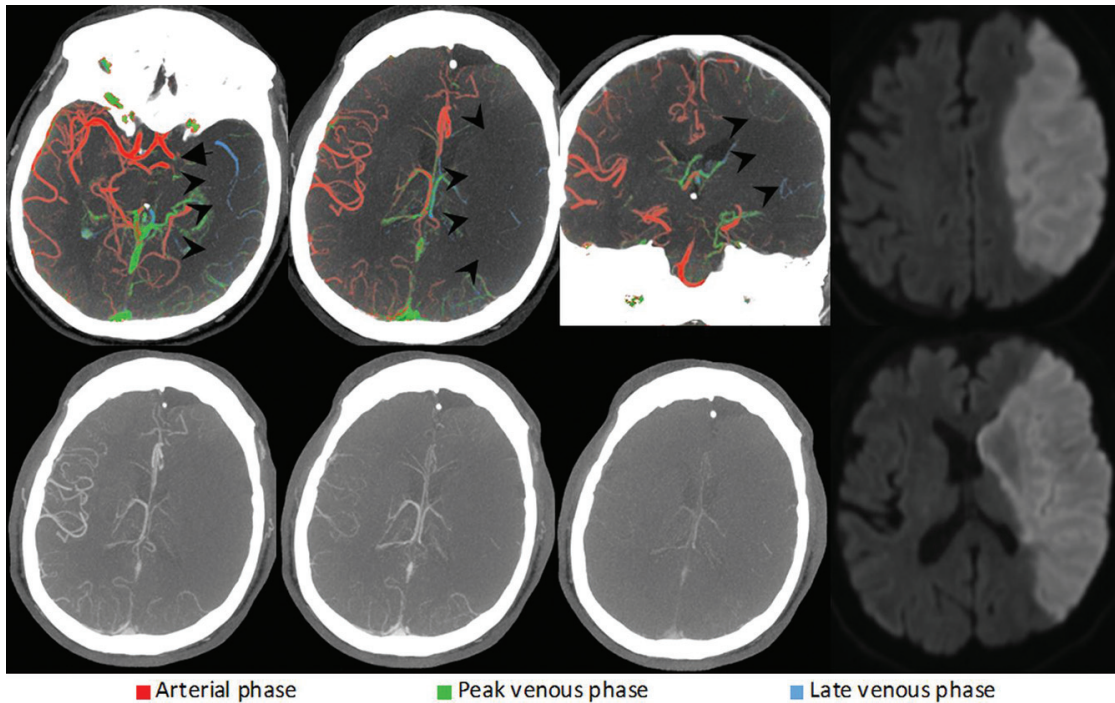

FIG 3. Left-sided ICA carotid L-occlusion (arrow). ColorViz summation maps (upper row) show a severely reduced vessel extent compared with the unaffected hemisphere (arrowheads). The few visible opacified vessels are predominantly blue, suggesting a 2-phase delay. This is consistent with poor pial artery filling and collateral flow. The patient was treated with antithrombotic therapy, and follow-up MR imaging after 24 hours (upper and lower right) shows complete infarction of the left MCA territory.

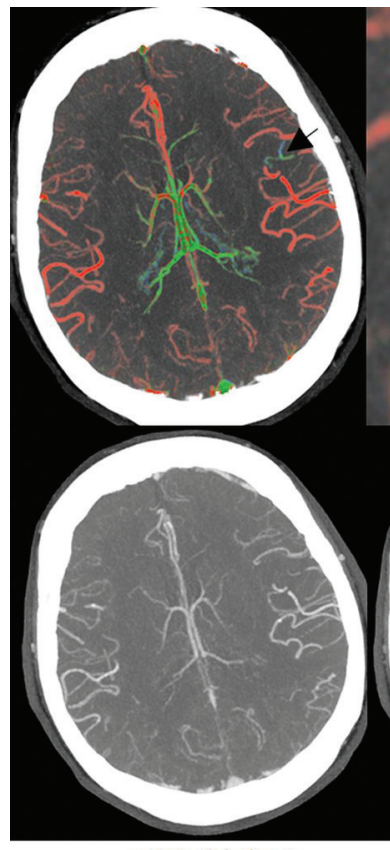

Arterial phase

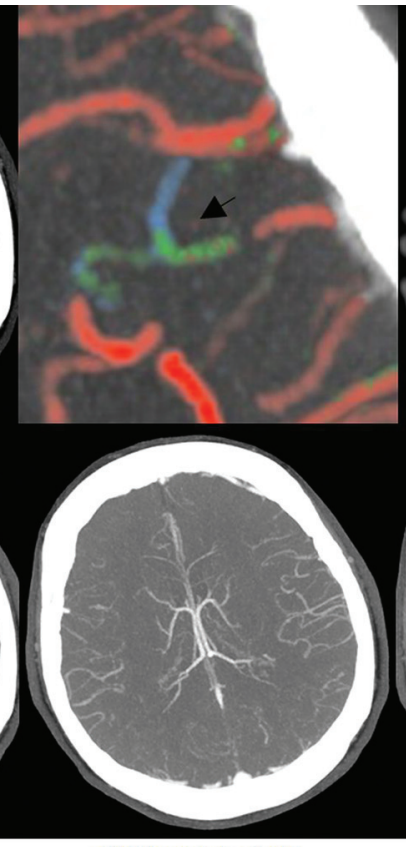

Peak venous phase

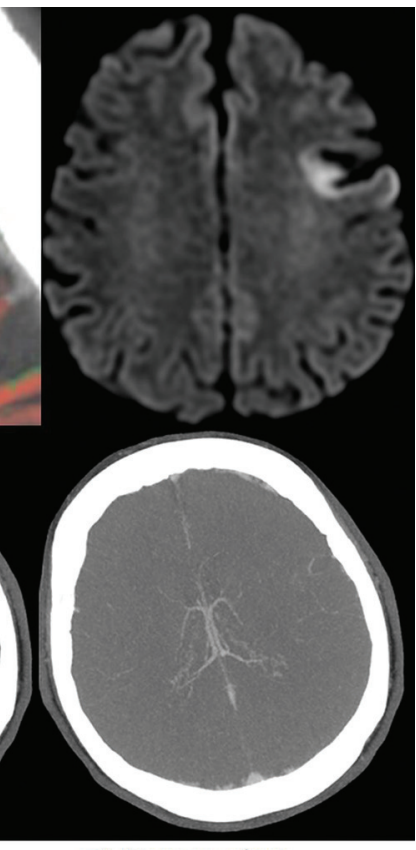

Late venous phase
FIG 4. Left-sided M4 segment MCA occlusion. ColorViz summation images (upper row) show a focal area with blue and green vessels in the left frontal lobe (black arrows), ie, an area with delayed pial artery filling and washout, suggestive of a distal vessel occlusion. The thrombus itself cannot be visualized; the occlusion was detected on the basis of arterial flow information only. Corresponding conventional MCTA images in the lower row also show a slight delay in left frontal pial artery filling and washout; however, the occlusion is not as easily appreciable as in the color-coded summation maps. The patient received intravenous alteplase. Follow-up MR imaging after 24 hours (upper right) shows an acute left frontal lobe infarction, thereby confirming the suspected site of intracranial occlusion. an advanced stroke CT imaging protocol (noncontrast CT, multiphase CTA, and CTP) in 1 single optimized, progressive workflow. The software fuses the vascular information from the different CTA phases (maximum of 9 phases) into a single color-coded view called ColorViz. It uses the original multiphase CTA thin slices to automatically display MIPs of the axial, coronal, sagittal, and oblique reformations. Colors are assigned on the basis of the timing and extent of contrast enhancement in the vessels and on a per-patient adaptive threshold technique; vessels maximally enhancing during the prevenous phase appear red; those that enhance in venous phase and venous phase are displayed in green and blue, respectively. The overall and phasespecific extent of pial en-hancement can then be quantified and compared with other territories in the contralateral hemisphere. Postprocessing is fully automated with the color-coded maps being available for review within 5-10 seconds. For evaluation of the vessel extent, the summation map can be switched to a single-color mode. Hence, the color display of the vasculature provides the full set of information about the dynamics and extent of pial artery filling from all mCTA phases in 1 single series. This feature expedites assessment of the intracranial vasculature compared with the conventional mCTA display format.

\section{Evaluating Pial Arterial Filling/ Collateral Flow}

mCTA is a reliable tool for assessment of pial artery filling in acute stroke imaging. Several studies (some singlecenter) have shown that it reduces interrater reliability and has excellent predictive validity with regard to clinical outcome. ${ }^{2,3}$ It has, therefore, been used in past ${ }^{4}$ and in ongoing clinical stroke trials such as ESCAPE NA1 and TEMPO 2. Pial artery filling is evaluated by assessing the extent of arterial filling and delay in that filling compared with the opposite side. A third metric is delayed washout of contrast within the pial arteries over the 3 


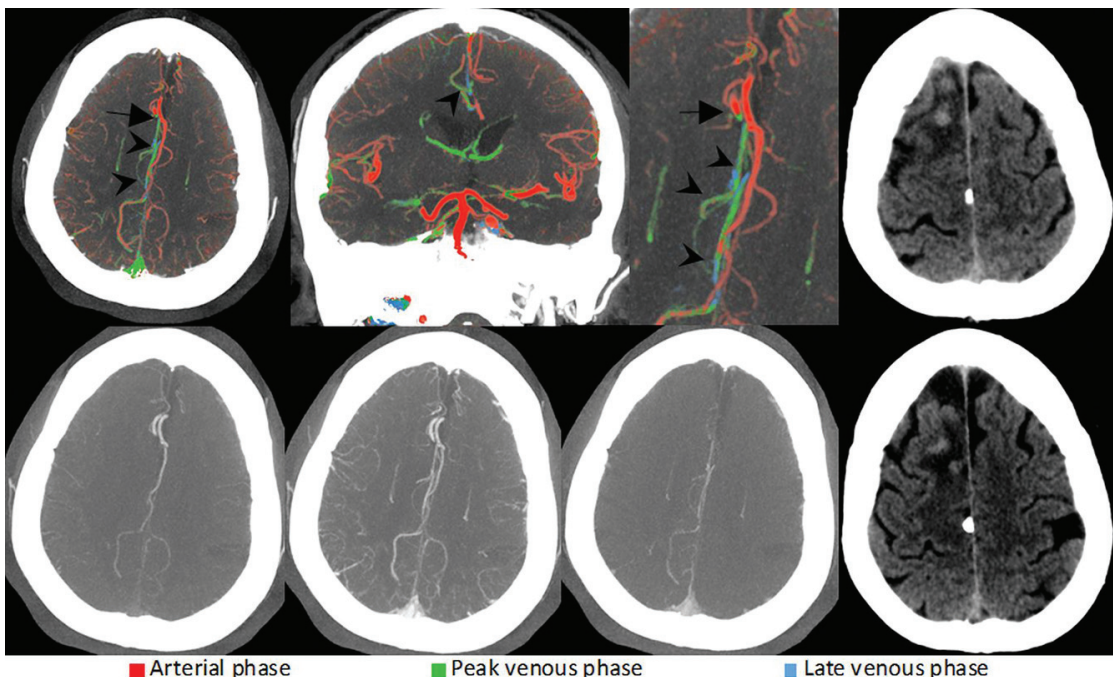

FIG 5. Right-sided A2 segment ACA occlusion (arrows). ColorViz summation images (upper row) show green and blue vessels distal to the occlusion (arrowheads), indicating impaired arterial filling within the right ACA territory. Corresponding conventional mCTA images are shown in the lower row. The patient received intravenous alteplase. Follow-up CT after 24 hours (upper and lower right) shows infarction in the right ACA territory.

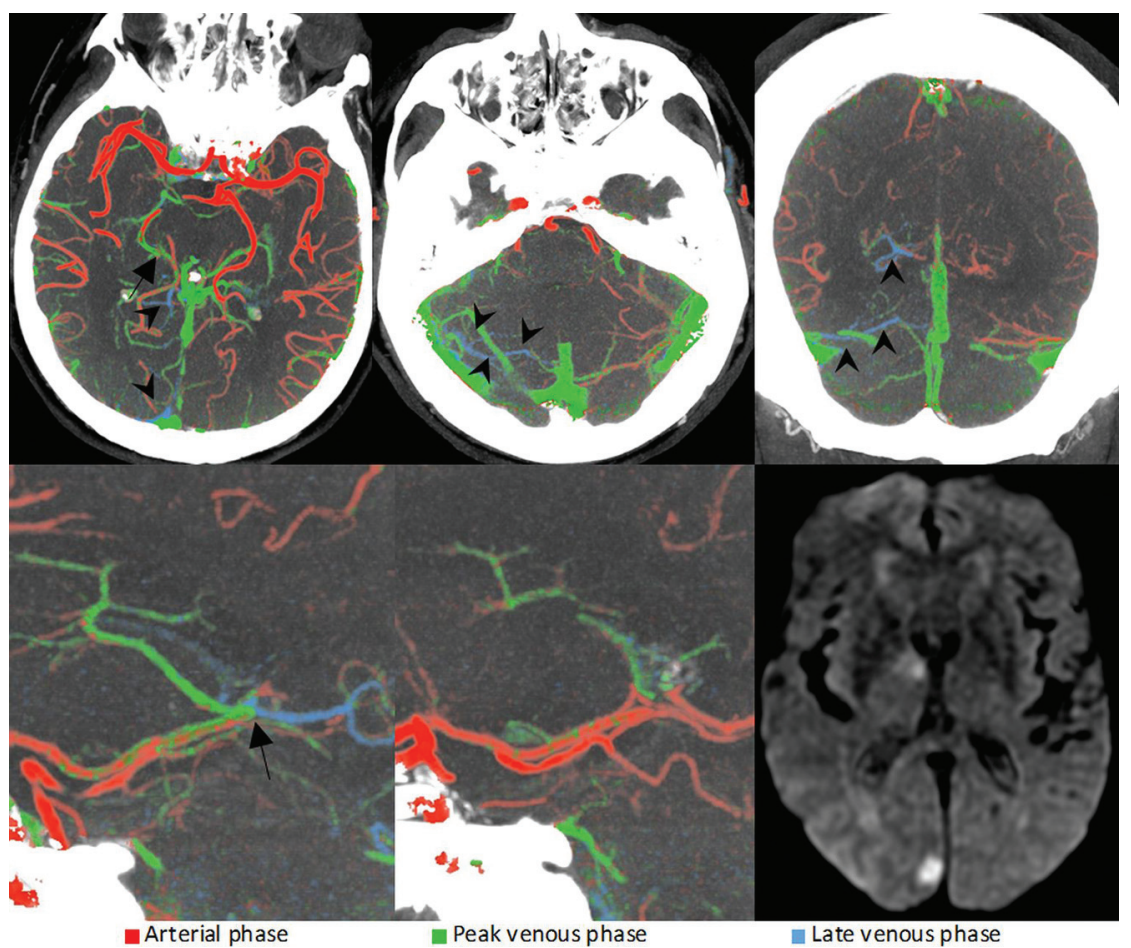

FIG 6. Right-sided fetal PCA occlusion (arrows). As opposed to the left PCA (lower middle image), the right distal PCA (lower left image) is blue in the color-coded summation images, suggestive of impaired filling of the distal vessel. The delayed arterial filling leads to a subsequent delay in venous filling in the posterior fossa on the affected side (arrowheads), a useful feature that is often encountered in posterior circulation occlusions and can help to identify the site of occlusion. Mechanical thrombectomy was performed. Follow-up MR imaging after 24 hours (lower right) reveals small right-sided occipital and thalamic infarct. phases. Although pial artery filling and collateral flow are relatively easy to assess on conventional mCTA, the color-coded summation maps (ColorViz) further facilitate interpretation by combining all information into a single image and using a color-coded display format. Image-interpretation time is a few seconds for colorcoded mCTA summation maps, as opposed to approximately 1 minute for conventional mCTA maps. An exemplary method for assessing collaterals on ColorViz based on assessing delay, extent, and washout within pial arteries is shown in Fig 1.

To assess pial artery filling using ColorViz, one visually determines the predominant vessel color (the color that is present in $>40 \%$ of the vessels) in the affected vascular territory. Under normal circumstances, predominantly red vessels (such as the vessels on the contralateral side in Figs 1-3) indicate no delay, predominantly green vessels indicate a 1-phase delay (Fig 2), and predominantly blue vessels, a 2-phase delay (Fig 3). The absence of visible vessels in the affected territory indicates "no filling." Because diseases such as congestive heart failure can lead to a reduction in cardiac output and systemic flow delays, the predominant color in the unaffected hemisphere is also assessed and compared with the predominant color in the affected side. Of note, this correction cannot be applied to conditions that cause unilateral flow delays, such as flow-limiting proximal ICA stenosis, a limitation of mCTA. The extent of pial artery filling is scored in a single color mode by comparing vessel extent in the affected territory with that in the contralateral hemisphere. Differentiation of veins and arteries is based on their distinct flow characteristics (veins are usually displayed in green or blue because they enhance in the peak venous and late venous phases), filling direction, morphology, and anatomic location. The affected hemisphere may be considered to have good collateral flow if pial vessels distal to the occlusion are well-seen and are predominantly red or green (Fig 1). 


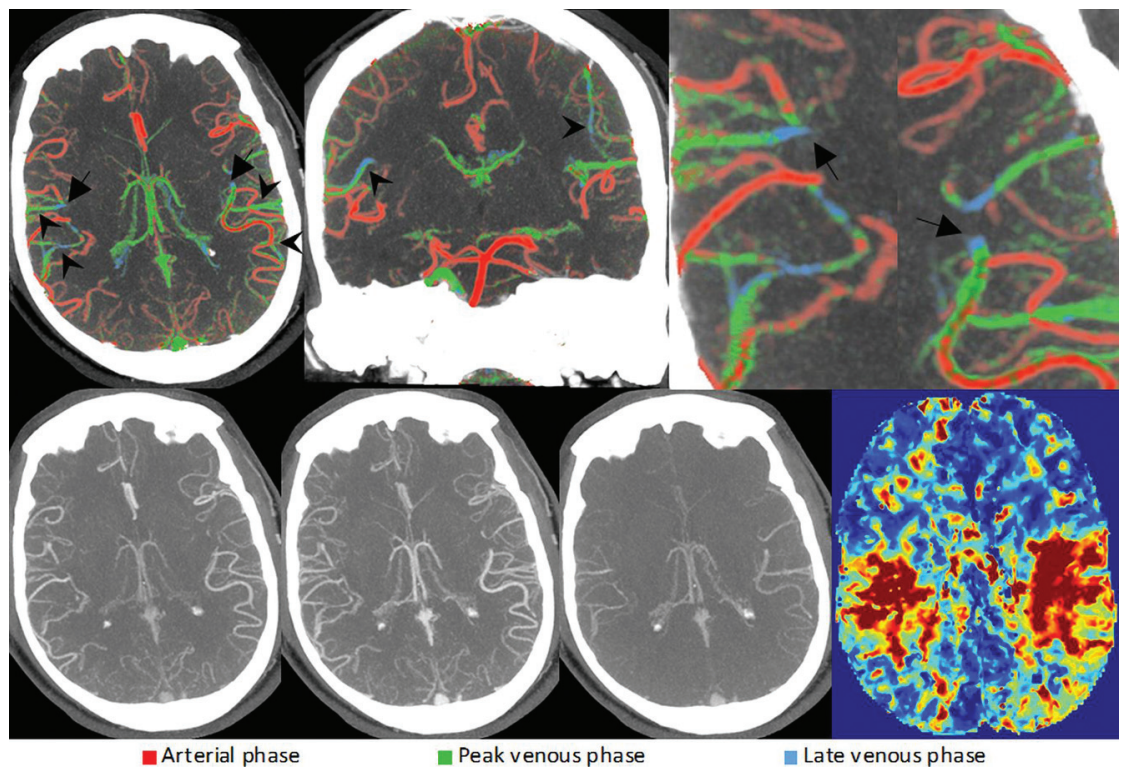

FIG 7. Bilateral M3 segment MCA occlusions (arrows) visualized through changes in the pial artery color compared with the surrounding pial arteries. Some cortical MCA branches of both hemispheres are depicted in green and blue (arrowheads), consistent with bilateral occlusions. Due to the symmetry of the occlusions, they are difficult to appreciate on conventional mCTA (lower row). CTP (lower right) shows prolonged time-to-maximum times in the affected territories, confirming the suspected occlusions. fully covered by the $4-, 8-$, or $10-\mathrm{cm}$ CTP slab that is frequently used with older generation scanners. Furthermore, mCTA does not succumb to skull base artifacts to the same extent as CTP and can therefore better visualize the posterior circulation. A particularly useful feature for posterior circulation occlusions is the venous filling delay in the posterior fossa, which results in a subsequent delay of venous filling, manifesting as an increased number of veins depicted in blue (Fig 6).

\section{Detection of Multiple Intracranial Thrombi}

Detecting intracranial thrombi other than the large-vessel occlusion that is easily identified is important because it may influence acute treatment strategies $^{8}$ and ultimately affect patient outcomes. ${ }^{9}$ Color-coded mCTA is able to detect multiple intracranial occlusions in either the same (Fig 7) or different (On-line Fig 1) vascular territories.
Intermediate pial artery filling and collateral flow are present if either pial artery extent, pial artery filling, or both are moderately reduced in the affected territory (Fig 2). Most frequently, intermediate collateral flow manifests as green vessels (indicating a 1phase delay) and reduced pial artery extent compared with the unaffected hemisphere.

Complete absence of pial artery filling or severely reduced extent and a 2-phase filling delay (ie, predominantly blue vessels) or complete absence of vessel opacification distal to the occlusion is considered poor pial artery filling (Fig 3).

\section{Increasing Sensitivity in Vessel Occlusion Detection}

While large-vessel occlusions are relatively easy to spot on singlephase CTA, distal occlusions are often missed. ${ }^{5,6}$ Both CTP (by detecting ischemia in the color maps) and mCTA (through delayed collateral filling and washout) have been shown to improve distal-vessel-occlusion detection compared with singlephase CTA alone. ${ }^{5-7}$ The additional color-indicator effect that is achieved using a color-coded display format and the ability to merge the vascular information in 1 rather than 3 series further facilitate detection of distal vessel occlusions on mCTA. The hallmark of distal occlusion in color-coded mCTA maps is areas of delayed flow manifesting as pial vessels in green and blue within 1 vascular territory (Fig 4).

Another advantage of color-coded mCTA summation maps is in detecting distal anterior cerebral artery (ACA) and posterior cerebral artery (PCA) occlusions (Figs 5 and 6). In theory, ACA and PCA occlusions can be easily detected on CTP by detecting perfusion abnormalities in the affected territories. However, the ACA and PCA territories are often not

\section{CONCLUSIONS}

Color-coded mCTA summation maps may facilitate easier assessment of acute stroke pathology, including better assessment of collateral status, distal occlusions, carotid pseudo-occlusions, intracranial stenosis, and thrombus permeability. ColorViz may be particularly useful for less experienced readers. Future work will, however, need detailed quantitative validation of each of the imaging constructs that ColorViz mCTA provides in patients with acute stroke.

Disclosures: Johanna M. Ospel—RELATED: Grant: Freiwillige Akademische Gesellschaft Basel, Julia-Bangerter-Rhyner Foundation, University of Basel Research Foundation, Comments: research scholarships. Ondrej VolnyRELATED: Grant: National Program of Sustainability II, Czech Republic, grant No. LQ1605.* Mayank Goyal-RELATED: Other: GE Healthcare, Comments: licensing agreement for systems of acute stroke diagnosis; UNRELATED: Consultancy: Medtronic, Stryker, MicroVention, Mentice, Comments: advice on acute stroke intervention; Grants/Grants Pending: Stryker, Comments: unrestricted research grant for the UNMASK-EVT study. * Bijoy K. Menon-OTHER RELATIONSHIPS: patent on systems of triage in acute stroke, stock ownership in Circle Neurovascular. *Money paid to the institution.

\section{REFERENCES}

1. de Havenon A, Mlynash M, Kim-Tenser MA, et al. Results from DEFUSE 3: good collaterals are associated with reduced ischemic core growth but not neurologic outcome. Stroke 2019;50:632-38 CrossRef Medline

2. Menon BK, d'Esterre CD, Qazi EM, et al. Multiphase CT angiography: a new tool for the imaging triage of patients with acute ischemic stroke. Radiology 2015;275:510-20 CrossRef Medline

3. Garcia-Tornel A, Carvalho V, Boned S, et al. Improving the evaluation of collateral circulation by multiphase computed tomography angiography in acute stroke patients treated with endovascular 
reperfusion therapies. Interv Neurol 2016;5:209-17 CrossRef Medline

4. Goyal M, Demchuk AM, Menon BK, et al. Randomized assessment of rapid endovascular treatment of ischemic stroke. $N$ Engl J Med 2015;372:1019-30 CrossRef Medline

5. Volny O, Cimflova P, Kadlecova P, et al. Single-phase versus multiphase CT angiography in middle cerebral artery clot detection-benefits for less experienced radiologists and neurologists. J Stroke Cerebrovasc Dis 2017;26:19-24 CrossRef Medline

6. Yu AY, Zerna C, Assis Z, et al. Multiphase CT angiography increases detection of anterior circulation intracranial occlusion. Neurology 2016;87:609-16 CrossRef Medline
7. Becks MJ, Manniesing R, Vister J, et al. Brain CT perfusion improves intracranial vessel occlusion detection on CT angiography. J Neuroradiol 2019;46:124-29 CrossRef Medline

8. Liebeskind DS, Bracard S, Guillemin F, et al. eTICI reperfusion: defining success in endovascular stroke therapy. J Neurointerv Surg 2019;11:433-38 CrossRef Medline

9. Ganesh A, Al-Ajlan FS, Sabiq F, et al. Infarct in a new territory after treatment administration in the ESCAPE Randomized Controlled Trial (Endovascular Treatment for Small Core and Anterior Circulation Proximal Occlusion with Emphasis on Minimizing CT to Recanalization Times). Stroke 2016;47:299398 CrossRef Medline 